\title{
A New Model for the Prediction of the Dog-bone Shape in Steel Mills
}

\author{
Duckjoong YUN, ${ }^{1)}$ Donghun LEE, ${ }^{1)}$ Jaeboo $\mathrm{KIM}^{21}$ and Sangmoo HWANG ${ }^{1) *}$ \\ 1) Department of Mechanical Engineering, Pohang University of Science and Technology, San 31, Hyoja-dong, Nam-gu, \\ Pohang, 790-784 Korea. E-mail: smhwang@postech.ac.kr \\ 2) Hyundai Heavy Industries Co., Jeonha 1-dong, Dong-gu, Ulsan, 682-792 Korea.
}

(Received on November 22, 2011; accepted on January 10, 2012)

\begin{abstract}
Precision control of the width of slabs, plates and strips, is vital for product quality and production economy in steel mills. A common practice in the production line is to perform vertical rolling and reduce the width in the roughing mill. However, the formation of so called 'dog-bone' at the edge of the slab would affect the final width after horizontal rolling that follows. Therefore, it is essential to predict and control the dog-bone shape. In this paper we present a new model for the prediction of the dog-bone shape during vertical rolling in roughing mills. The model is developed on the basis of the minimum energy principle for a rigid-plastic material and a three-dimensional admissible velocity field. The predictions accuracy of the proposed model is examined via comparison with predictions from finite element simulation and also with experimental data.
\end{abstract}

KEY WORDS: vertical rolling; finite element method; dog-bone shape; minimum energy principle; admissible velocity field.

\section{Introduction}

Roughing rolling, conducted in plate mills and hot strip mills, is to reduce the thickness of the slabs prior to finish rolling. In addition to that, simultaneously performed during roughing rolling, in general, is to reduce the width of the slabs so as to control the width of the product as desired. While the thickness reduction is achieved by horizontal rolling, the width reduction is achieved by vertical rolling, or edge rolling, as illustrated in Fig. 1. During vertical rolling, the slab is compressed in the width direction, and as a result, partial thickening occurs near its edges, which is known as dog-bone shape, as illustrated in Fig. 2. The formation of the dog-bone gravely affects the final width after horizontal rolling that follows, and therefore, its prediction is vital for sound process control.

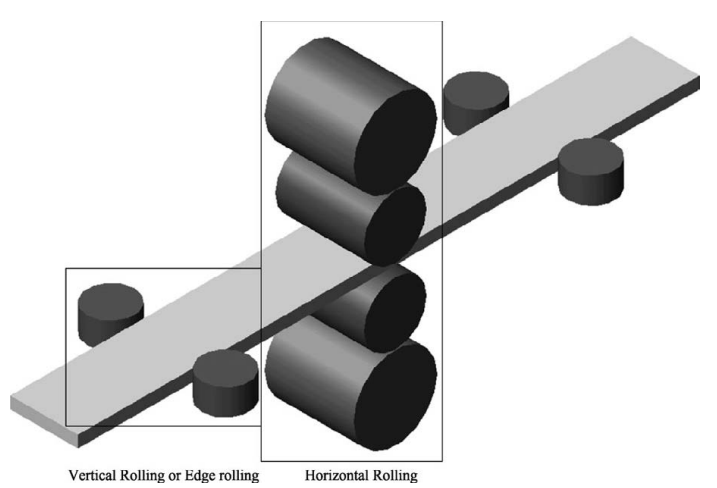

Fig. 1. Vertical rolling (Edge rolling) and horizontal rolling in a roughing mill.
In the past, several models were proposed for the prediction of the dog-bone shape. ${ }^{1-5)}$ Tazoe, ${ }^{1)}$ et al. derived a mathematical formula based on the experimental data obtained by Shibahara, et $a l .{ }^{3)}$ Later, it was modified by Ginzburg. ${ }^{2}$ Those formulas were expressed in terms of the dimensions of the material to be rolled and roll geometry. Okado, et al. ${ }^{4)}$ conducted a series of plasticine experiments in laboratoryscale mills, from which they came up with a model in which

Initial cross-sectional area of the slab

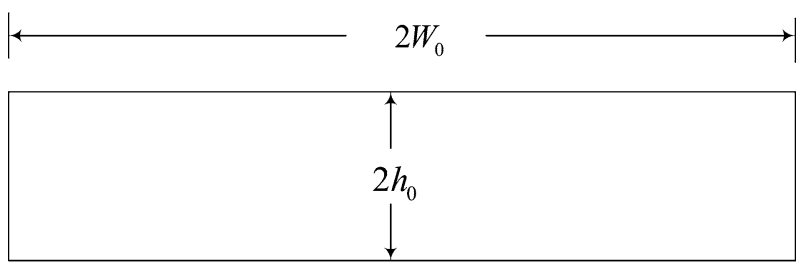

After vertical rolling

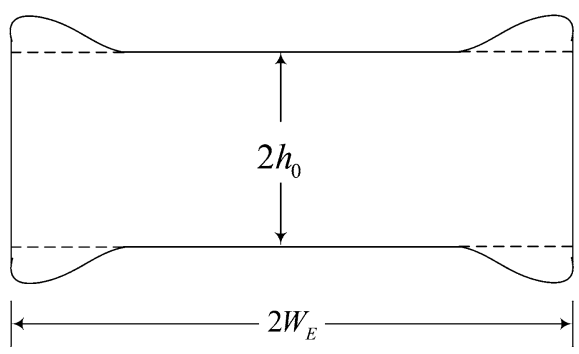

Fig. 2. The cross-sectional shape of the slab before and after edge rolling. 
the dog-bone shape is defined by four main parameters, each of which is given in terms of initial thickness of the slab and the amount of width reduction. However, these formulas are empirical, yet few attempts have been made to predict the dog-bone shape on the basis of the theory of plasticity.

We feel that in order to precisely predict the slab width in a roughing mill, the model for the prediction of slab width after horizontal rolling should be capable of precisely taking into account the effect of edge rolling that preceded horizontal rolling, or, more precisely, the effect of the detailed aspect of the dog-bone profile. It is in this regard that we believe it is essential to develop first a model which can precisely predict the dog-bone profile.

The finite element method may be the best choice for this purpose. However, it is not suitable for on-line prediction and control, due to the fact that its application would require consumption of excessive computation time. For instance, finite element simulation of three-dimensional deformation of a slab in rolling, using a steady-state formulation, may easily take several hours of computation time, and much more if a non-steady formulation is employed. Instead, the approach adopted in this paper is one based on the minimum energy principle for a rigid-plastic material, combined with an elegant three dimensional admissible velocity field.

Available are the velocity field proposed by researchers ${ }^{6-8)}$ in association with the application of the minimum energy principle to the analysis of plastic deformation of horizontal rolling and shape rolling. Unfortunately, they are hardly applicable to vertical rolling, due to its extreme nature of deformation inhomogeneity, and it is in this regard this paper presents perhaps for the first time an admissible velocity field for the analysis of edge rolling. The prediction accuracy of the proposed model is examined via comparison with predictions from finite element simulation and also with experimental data.

\section{Minimum Energy Principle (Upper Bound Theorem)}

A minimum energy principle as applied to a rigid-plastic material states that for all admissible velocity fields $u_{i}^{*}$, the actual field $u_{i}$ minimizes

$$
\phi\left(u_{i}^{*}\right)=\int_{\Omega} \dot{\sigma}^{*} d \Omega+\int_{\Gamma_{C}} \lambda\left|\vec{u}_{t}^{*}-\vec{u}_{t D}\right| d \Gamma_{C}+\sum_{l s=1}^{n-1} \int_{\Gamma_{l s}} k\left|\overrightarrow{\Delta u}^{*}\right| d \Gamma_{l_{s}}
$$

As shown in Fig. 3, a slab is rolled through a pair of vertical flat rolls of radius $R$, and slab's width is reduced from $2 W_{0}$ to $2 W_{E}$ The axes $x, y$, and $z$ are chosen so as to represent the rolling direction, vertical direction, and the direction across the slab, respectively. Note that, in the bite zone, the thickness of the slab may be represented by $2 h=2 h(x, z)$. Thus, the inlet thickness $2 h_{0}$ changes into $2 h(l, z)$ after rolling, where $l$ is the projected length of the roll-slab contact arc.

Then, the first term of Eq. (1), which is the energy dissipation due to plastic deformation, may be calculated from

$$
E_{p}=\int_{0}^{l} \int_{0}^{W(x)} \int_{0}^{h(x, z)} \bar{\sigma}(\bar{\varepsilon}, \dot{\bar{\varepsilon}}, T) \dot{\bar{\varepsilon}}^{*} d y d z d x
$$

It is to be noted that $\bar{\sigma}(\bar{\varepsilon}, \dot{\bar{\varepsilon}}, T)$, which may be calculated from the flow stress curves, for example, obtained by Shida, ${ }^{11)}$ is not known since $\bar{\varepsilon}$ and $\dot{\bar{\varepsilon}}$, which represent actual effective strain distribution and actual effective strain rate distri-

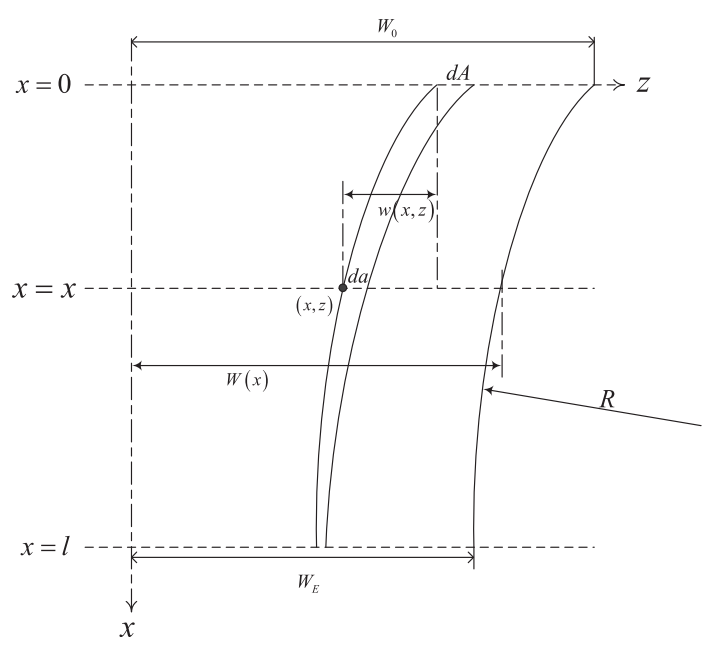

Fig. 3. Definition sketch of the bite zone in edge rolling.

bution in the bite zone, are not known.

$\bar{\varepsilon}$ may closely be approximated by the effective strain distribution predicted by 2-D elementary rolling theory when calculating $\bar{\sigma}$, or

$$
\bar{\varepsilon} \approx-\frac{2}{\sqrt{3}} \ln \frac{W(x)}{W_{0}}
$$

Also, assuming that the admissible velocity field minimizing $\phi\left(u_{i}^{*}\right)$ under the condition $\dot{\bar{\varepsilon}}=\dot{\bar{\varepsilon}}^{*}$ may be sufficiently close to the actual velocity field, we approximate $\dot{\bar{\varepsilon}}$ by

$$
\dot{\bar{\varepsilon}} \approx \dot{\bar{\varepsilon}}^{*}
$$

The second term, which is the energy dissipation due to friction, may be calculated from

$$
E_{f}=\int_{0}^{l} \int_{0}^{h(x, W(x))} \mu \sigma_{n}\left|\vec{u}_{t}^{*}-\vec{u}_{t D}\right| d y d x
$$

It is assumed that the frictional stress is constant over the roll-slab contact surface, or, $\sigma_{n}=F / l$, where $F$ denotes the roll force per unit slab thickness.

The magnitude of velocity of the slab relative to the roll at the roll-slab contact surface is given by

$$
\left|\vec{u}_{t}^{*}-\vec{u}_{t D}\right|=\sqrt{\left(u_{x}^{*}-u_{t D} \cos \theta\right)^{2}+\left(u_{y}^{*}\right)^{2}+\left(u_{z}^{*}-u_{t D} \sqrt{1-\cos ^{2} \theta}\right)^{2}}
$$

where

$$
\cos \theta=\frac{\sqrt{R^{2}-(x-l)^{2}}}{R}
$$

The third term, which is the energy dissipation due to velocity discontinuity at the roll entry and at the roll exit, becomes

$$
\begin{aligned}
E_{d} & =\left.\int_{0}^{W_{0}} \int_{0}^{h_{0}} k \sqrt{\left(u_{y}^{*}\right)^{2}+\left(u_{z}^{*}\right)^{2}} d y d z\right|_{x=0} \\
& +\left.\int_{0}^{W_{E}} \int_{0}^{h(l, z)} k \sqrt{\left(u_{y}^{*}\right)^{2}+\left(u_{z}^{*}\right)^{2}} d y d z\right|_{x=l}
\end{aligned}
$$

where $k=\bar{\sigma} / \sqrt{3}$

In order to derive an admissible field we consider the lateral strain defined by 


$$
\frac{d w}{d z}=\frac{d a-d A}{d A}
$$

where $d a-d A$ denotes the change in the length of an infinitesimal segment, and $w=w(x, z)$ denotes the lateral displacement, as shown in Fig. 3.

From the incompressibility condition,

$$
u_{x}(x, z)=\frac{V_{0} \cdot h_{0} \cdot d A}{h(x, z) \cdot d a}
$$

where $V_{0}$ is inlet velocity of the slab.

Substituting Eq. (9) into Eq. (10) yields

$$
u_{x}(x, z)=\frac{V_{0} \cdot h_{0}}{h(x, z)} \cdot\left(1-\frac{d w}{d z}\right)
$$

We also note that

$$
\frac{u_{z}(x, z)}{u_{x}(x, z)}=\frac{d w(x, z)}{d x}
$$

From which we obtain

$$
u_{z}(x, z)=\frac{V_{0} \cdot h_{0}}{h(x, z)} \cdot\left(1-\frac{d w}{d z}\right) \cdot \frac{d w}{d x}
$$

Substituting Eqs. (11) and (13) into the incompressibility condition

$$
\frac{\partial u_{y}}{\partial y}=-\frac{\partial u_{x}}{\partial x}(x, z)-\frac{\partial u_{z}}{\partial z}(x, z)
$$

and using the boundary condition $u_{y}=0$ at $y=0$, we obtain

$$
\begin{aligned}
\frac{u_{y}(x, y, z)}{V_{0} \cdot h_{0}}= & {\left[( \frac { \partial w } { \partial z } - 1 ) \cdot \left\{\frac{\partial}{\partial x}\left(\frac{1}{h(x, z)}\right)+\frac{\partial}{\partial z}\left(\frac{1}{h(x, z)}\right) \cdot \frac{\partial w}{\partial x}\right.\right.} \\
& \left.\left.+\frac{1}{h(x, z)} \frac{\partial^{2} w}{\partial x \partial z}\right\}+\frac{1}{h(x, z)} \cdot\left(\frac{\partial^{2} w}{\partial z^{2}} \cdot \frac{\partial w}{\partial x}+\frac{\partial^{2} w}{\partial x \partial z}\right)\right] \cdot y
\end{aligned}
$$

\section{Mathematical Representation of the Dog-bone Shape}

The admissible velocity field derived above has two unknown functions. That is, $h(x, z)$, which may be called the dog-bone shape function, and the lateral displacement field $w(x, z)$. In order to describe $h(x, z)$ we may divide the bite zone into two zones, as shown in Fig. 4.

zone I $(0<z<W(x)-A)$ : the stem part of the dog bone

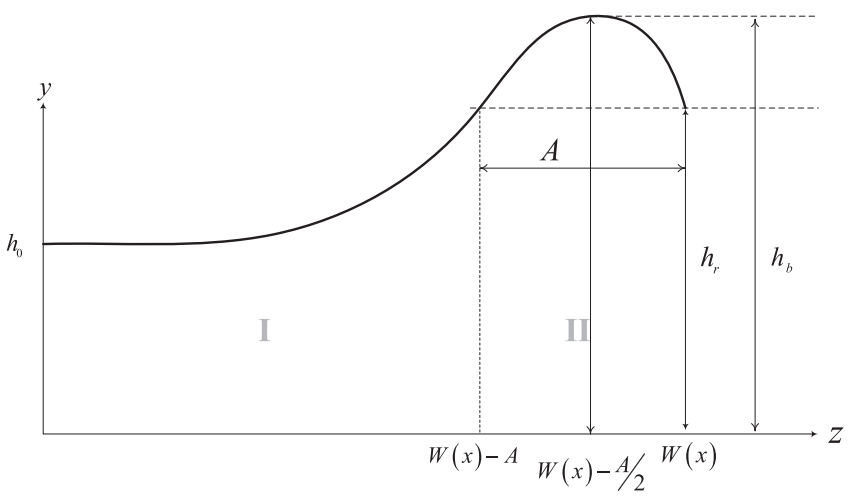

Fig. 4. Definition of zone I and zone II of the dog-bone profile, at roll exit. zone II $W(x)-A<z<W(x))$ : the end part of the dog-bone, which is round.

Where $A$ is a constant and $W(x)$ is the slab width in the bite zone at $x$, which may be given by (see Fig. 3 )

$$
W(x)=W_{E}+R-\sqrt{R^{2}-(x-l)^{2}}
$$

Noting that the dog-bone would increase both along $x$ and $z$ direction, we may propose the dog-bone function for zone I as follows:

$$
h_{1}(x, z)=K_{0}+K_{1} q(x) z^{\alpha} .
$$

where $K_{0}, K_{1}$ and $\alpha$ are constants, and

$$
q(x)=W_{0}-W(x) \text {. }
$$

Also, we may propose the dog-bone function for the end part as follows:

$$
\begin{aligned}
h_{2}(x, z) & =K_{2}+q(x)\left[K_{3}(x)\left\{z-W(x)+\frac{A}{2}\right\}^{4}\right. \\
& \left.+K_{4}(x)\left\{z-W(x)+\frac{A}{2}\right\}^{2}+K_{5}\right]
\end{aligned}
$$

where constants $K_{2}$ and $K_{5}$, as well as functions $K_{3}(x), K_{4}(x)$ are to be determined.

Note that at the end part the shape is symmetric with respect to the line $z=W(x)-\frac{A}{2}$. Process simulation with a three dimensional rigid-viscoplastic finite element method ${ }^{9)}$ reveals that this line is a close approximation of the line along which the peak in the dog-bone profile appears in the bite zone, as shown in Fig. 5.

It is clear that we have in total eight constants and functions $\left(A, K_{1}, \alpha\right)$ that have to be determined. On the other hand, the conditions to be satisfied are, from Fig. 4,

$$
\begin{array}{r}
h_{1}(0, z)=h_{0} \ldots \ldots \\
h_{1}\left(l, W_{E}-A\right)=h_{r} \ldots \\
h_{2}(0, z)=h_{0} \ldots \ldots . \\
h_{2}\left(l, W_{E}-\frac{A}{2}\right)=h_{b}
\end{array}
$$

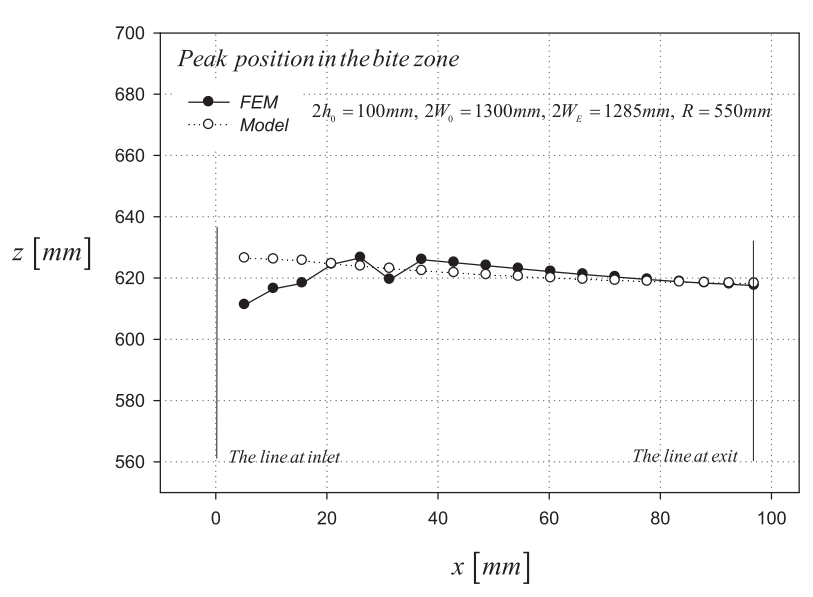

Fig. 5. The line along which the peak in the dog-bone profile appears in the bite zone. Predictions from FEM and the present model. 


$$
\begin{aligned}
h_{1}(x, W(x)-A) & =h_{2}(x, W(x)-A) \ldots \ldots . . \\
\left.\frac{\partial h_{1}(x, z)}{\partial z}\right|_{z=W(x)-A} & =\left.\frac{\partial h_{2}(x, z)}{\partial z}\right|_{z=W(x)-A}
\end{aligned}
$$

where $h_{0}$ is the inlet thickness, $h_{b}$ is the maximum height of the dog-bone, and $h_{r}$ is the height of the dog-bone at the edge of the slab. Note that both $h_{b}$ and $h_{r}$ denote the values at the roll exit.

From Eqs. (20), (21), (22), and (23), we obtain

$$
\begin{array}{r}
K_{0}=h_{0} \ldots \ldots \ldots \ldots \\
K_{1}=\frac{h_{r}-h_{0}}{q(l)\left(W_{E}-A\right)^{\alpha}} \\
K_{2}=h_{0} \ldots \ldots \ldots \ldots \\
K_{5}=\frac{h_{b}-h_{0}}{q(l)} \ldots \ldots
\end{array}
$$

Also, from Eqs. (24) and (25), we obtain

$$
\begin{aligned}
K_{3}(x) & =\left(\frac{\left(h_{b}-h_{0}\right)}{q(l)}-\left(h_{r}-h_{0}\right) \frac{(W(x)-A)^{\alpha}}{q(l)\left(W_{E}-A\right)^{\alpha}}\right)\left(\frac{A}{2}\right)^{-4} \\
& -\left(\frac{\alpha\left(h_{r}-h_{0}\right)}{2} \frac{(W(x)-A)^{\alpha-1}}{q(l)\left(W_{E}-A\right)^{\alpha}}\right)\left(\frac{A}{2}\right)^{-3} \\
K_{4}(x) & =2\left(\left(h_{r}-h_{0}\right) \frac{(W(x)-A)^{\alpha}}{q(l)\left(W_{E}-A\right)^{\alpha}}-\frac{\left(h_{b}-h_{0}\right)}{q(l)}\right)\left(\frac{A}{2}\right)^{-2} \\
& +\left(\frac{\alpha\left(h_{r}-h_{0}\right)}{2} \frac{(W(x)-A)^{\alpha-1}}{q(l)\left(W_{E}-A\right)^{\alpha}}\right)\left(\frac{A}{2}\right)^{-1}
\end{aligned}
$$

\section{Determination of the Lateral Displacement Field}

For the calculation for the lateral displacement, we may assume that $u_{x}(x, z)$ appearing in Eq. (11) may be replaced by $V_{0}$. Finite element simulation reveals that the approximation is acceptable, with the maximum error being $3 \%$ for the case investigated, as shown in Fig. 6. Then, from Eq. (11) we obtain

$$
w(x, z)=\int_{0}^{z}\left\{1-\frac{h(x, z)}{h_{0}}\right\} d z
$$

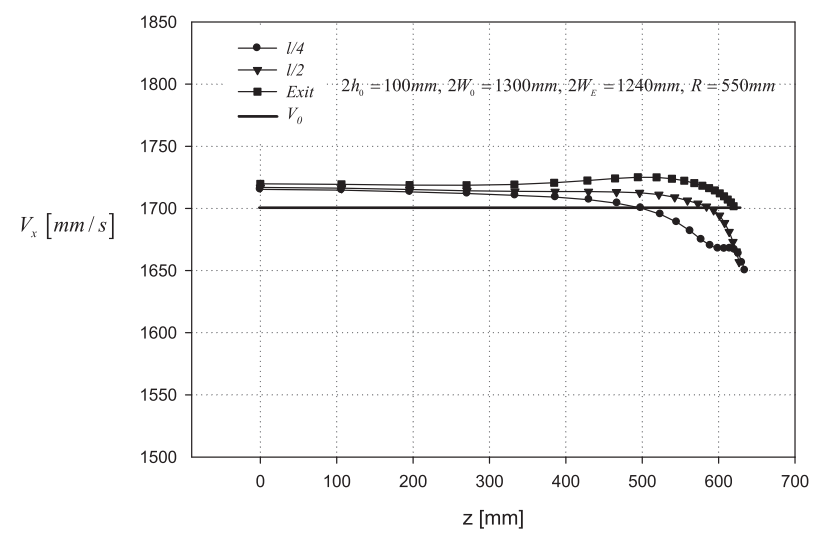

Fig. 6. The distribution of $u_{x}(x, z)$ in the bite zone, predicted by FEM. Inlet velocity of the slab $=V_{0}$.
It follows that, for zone I

$$
w_{1}(x, z)=-\frac{1}{\alpha+1} \frac{h_{r}-h_{0}}{h_{0}} \frac{1}{\left(W_{E}-A\right)^{\alpha}} \frac{q(x)}{q(l)} z^{\alpha+1} \ldots
$$

And for zone II

$$
\begin{aligned}
w_{2}(x, z)= & z-\frac{K_{2}+q(x) K_{5}}{h_{0}} z \\
& -\frac{q(x) K_{3}(x)}{h_{0}}\left[\frac{1}{5} z^{5}-\left\{W(x)-\frac{A}{2}\right\} z^{4}+2\left\{W(x)-\frac{A}{2}\right\}^{2} z^{3}\right. \\
& \left.-2\left\{W(x)-\frac{A}{2}\right\}^{3} z^{2}+\left\{W(x)-\frac{A}{2}\right\}^{4} z\right] \\
& -\frac{q(x) K_{4}(x)}{h_{0}}\left[\frac{1}{3} z^{3}-\left\{W(x)-\frac{A}{2}\right\} z^{2}+\left\{W(x)-\frac{A}{2}\right\}^{2} z\right] \\
& +C_{2}(x)
\end{aligned}
$$

From the boundary condition $w_{1}(x, W(x)-A)=w_{2}(x, W(x)-$ $A$ ) it may be shown that

$$
\begin{aligned}
C_{2}(x)= & -\frac{1}{\alpha+1} \frac{h_{r}-h_{0}}{h_{0}} \frac{1}{\left(W_{E}-A\right)^{\alpha}} \frac{q(x)}{q(l)}(W(x)-A)^{\alpha+1} \\
& -\left(1-\frac{K_{2}+q(x) K_{5}}{h_{0}}\right)(W(x)-A) \\
& +\frac{q(x) K_{3}(x)}{h_{0}}\left[\frac{1}{5}\{W(x)-A\}^{5}-\left\{W(x)-\frac{A}{2}\right\}\{W(x)-A\}^{4}\right. \\
& +2\left\{W(x)-\frac{A}{2}\right\}^{2}\{W(x)-A\}^{3} \\
& -2\left\{W(x)-\frac{A}{2}\right\}^{3}\{W(x)-A\}^{2} \\
& \left.+\left\{W(x)-\frac{A}{2}\right\}^{4}\{W(x)-A\}\right] \\
& +\frac{q(x) K_{4}(x)}{h_{0}}\left[\frac{1}{3}\{W(x)-A\}^{3}-\left\{W(x)-\frac{A}{2}\right\}\{W(x)-A\}^{2}\right. \\
& \left.+\left\{W(x)-\frac{A}{2}\right\}^{2}\{W(x)-A\}\right]
\end{aligned}
$$

Also, from the condition $w_{2}\left(l, W_{E}\right)=W_{E}-W_{0}$ it may be shown that

$$
h_{b}=\frac{I_{6}\left(I_{1}-I_{3}\right)+I_{7}\left(I_{4}-I_{2}\right)+h_{0} I_{5}}{\left\{\frac{I_{1}-I_{3}}{\left(\frac{A}{2}\right)^{4}}+\frac{2 I_{4}-2 I_{2}}{\left(\frac{A}{2}\right)^{2}}+A\right\}}
$$

where

$$
\begin{aligned}
I_{1}= & \frac{1}{5} W_{E}^{5}-\left(W_{E}-\frac{A}{2}\right) W_{E}^{4}+2\left(W_{E}-\frac{A}{2}\right)^{2} W_{E}^{3} \\
& -2\left(W_{E}-\frac{A}{2}\right)^{3} W_{E}^{2}+\left(W_{E}-\frac{A}{2}\right)^{4} W_{E}
\end{aligned}
$$




$$
\begin{aligned}
& I_{2}=\frac{1}{3} W_{E}^{3}-\frac{A}{2} W_{E}^{2}+\frac{A^{2}}{4} W_{E} \\
& I_{3}=\frac{1}{5}\left(W_{E}-A\right)^{5}-\left(W_{E}-\frac{A}{2}\right)\left(W_{E}-A\right)^{4}+2\left(W_{E}-\frac{A}{2}\right)^{2}\left(W_{E}-A\right)^{3} \\
& -2\left(W_{E}-\frac{A}{2}\right)^{3}\left(W_{E}-A\right)^{2}+\left(W_{E}-\frac{A}{2}\right)^{4}\left(W_{E}-A\right) \\
& I_{4}=\frac{1}{3}\left(W_{E}-A\right)^{3}-\left(W_{E}-\frac{A}{2}\right)\left(W_{E}-A\right)^{2}+\left(W_{E}-\frac{A}{2}\right)^{2}\left(W_{E}-A\right) \\
& I_{5}=-\frac{1}{\alpha+1} \frac{h_{r}-h_{0}}{h_{0}}\left(W_{E}-A\right)+A-\left(W_{E}-W_{0}\right) \ldots \\
& I_{6}=\frac{h_{r}+\alpha\left(\frac{h_{r}-h_{0}}{W_{E}-A}\right)\left(\frac{A}{4}\right)}{\left(\frac{A}{2}\right)^{4}} \\
& I_{7}=\frac{2 h_{r}+\alpha\left(\frac{h_{r}-h_{0}}{W_{E}-A}\right)\left(\frac{A}{4}\right)}{\left(\frac{A}{2}\right)^{2}}
\end{aligned}
$$

\section{Determination of the Parameter $A$}

Assuming that the material is plain carbon steel, a mathematical expression describing the effect of the rolling conditions on the parameter $A$ may be given by

$$
A=f\left(\mu, h_{0}, W_{0}, W_{E}, \omega, T, C_{0}, R\right) .
$$

where $R$ and $\omega$ denote the roll radius and the roll angular velocity, respectively, and $T$ and $C_{0}$ denote the slab temperature and the carbon contents, respectively.

Assuming that the coefficient of friction is given, Eq. (44) may be written as, in a dimensionless form,

$$
\frac{A}{W_{0}}=f\left(r, \frac{h_{0}}{W_{0}}, \frac{h_{0}}{R}, \frac{T}{c_{1}}, \frac{\omega}{c_{2}}, C_{0}\right)
$$

where $c_{1}$ and $c_{2}$ are proper units introduced to render $T$ and $\omega$ dimensionless, and

$$
r=\frac{W_{0}-W_{E}}{W_{0}}
$$

Using the three dimensional finite element code developed for the prediction of plastic deformation occurring during rolling, ${ }^{9)}$ a series of FE simulation is performed to investi-

\begin{tabular}{|c|c|c|}
\hline \multicolumn{3}{|c|}{ Process conditions } \\
\hline $\bar{\sigma}$ & $\mathrm{kN} / \mathrm{mm}^{2}$ & $\begin{array}{l}\text { flow stress for } 0.01 \% \sim 1.2 \% \text { carbon steel, } \\
\text { see Reference [11] }\end{array}$ \\
\hline$r=\frac{W_{0}-W_{E}}{W_{0}}$ & & $0.018-0.04$ \\
\hline$h_{0} / W_{0}$ & & $0.03125-0.2174$ \\
\hline$h_{0} / R$ & & $0.042-0.2273$ \\
\hline$T / c_{1}$ & & $800-1200$ \\
\hline$\omega / c_{2}$ & & $2-6$ \\
\hline$\mu$ & & 0.3 \\
\hline $2 h_{0}$ & $m m$ & $50-250$ \\
\hline $2 W_{0}$ & $m m$ & $1150-1600$ \\
\hline$R$ & $m m$ & $300-600$ \\
\hline$T$ & ${ }^{\circ} \mathrm{C}$ & $800-1200$ \\
\hline$\omega$ & $\mathrm{rad} / \mathrm{s}$ & $2-6$ \\
\hline \multicolumn{3}{|c|}{ Total number of sets : 154} \\
\hline
\end{tabular}
gate the effect of each of the variables in Eq. (45). The rolling conditions selected for this investigation are summarized in Table 1.

A detailed aspect of the formation of the dog-bone due to edge rolling, as predicted by FE simulation, is illustrated in Fig. 7. It is revealed by the finite element simulation that $\frac{A}{W_{0}}$ increases with the increase of $r$ and $\frac{h_{0}}{W_{0}}$, while it is
Table 1. Process conditions for FE simulation of deformation in edge rolling.

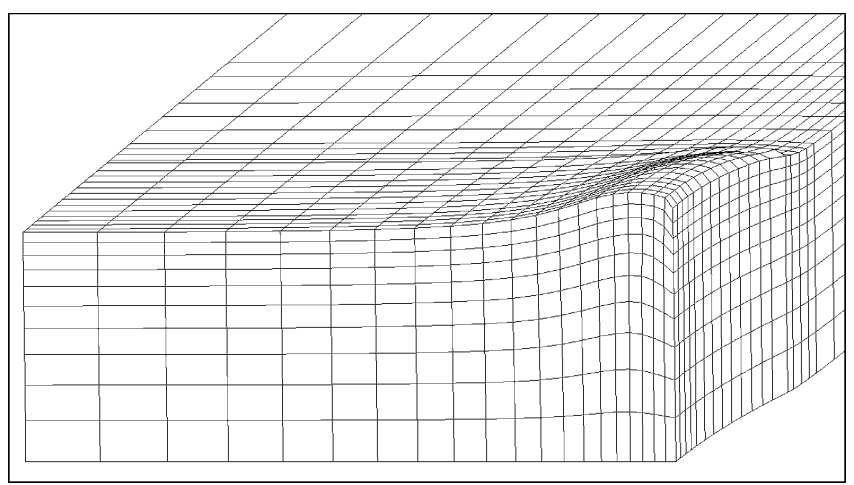

Fig. 7. Formation of the dog-bone due to edge rolling, as predicted by FEM.

decreased with the increase of $\frac{h_{0}}{R}$, as shown in Figs. 8-10. Also revealed is that $\frac{A}{W_{0}}$ is little affected by the change of $\frac{T}{c_{1}}, \frac{\omega}{c_{2}}$ and $C_{0}$, implying that the effect of the flow stress characteristics of the material on the formation of the dogbone may be negligible.

A mathematical formula may be derived from the regression of the data obtained from a series of FE simulation. The result is, for $\mu=0.3$

$$
\begin{aligned}
\frac{A}{W_{0}}= & 0.057-0.256 \frac{h_{0}}{R}+0.496 \frac{h_{0}}{W_{0}}+0.437 r-1.036 \frac{h_{0}}{W_{0}} \frac{h_{0}}{R} \\
& +7.441 r \frac{h_{0}}{R}+22.16 r \frac{h_{0}}{W_{0}}+8.233 r^{2}-61.46 r \frac{h_{0}}{W_{0}} \frac{h_{0}}{R} \\
& -290.76 r^{2} \frac{h_{0}}{R}-90.86 r^{2} \frac{h_{0}}{W_{0}}+942.16 r^{2} \frac{h_{0}}{W_{0}} \frac{h_{0}}{R}
\end{aligned}
$$




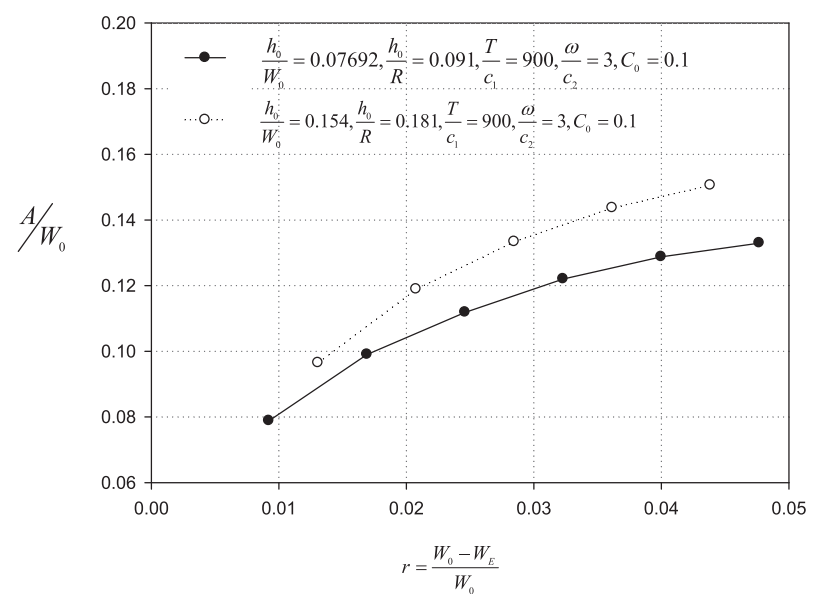

Fig. 8. The effect of $\mathrm{r}$ on $\frac{A}{W_{0}}$.

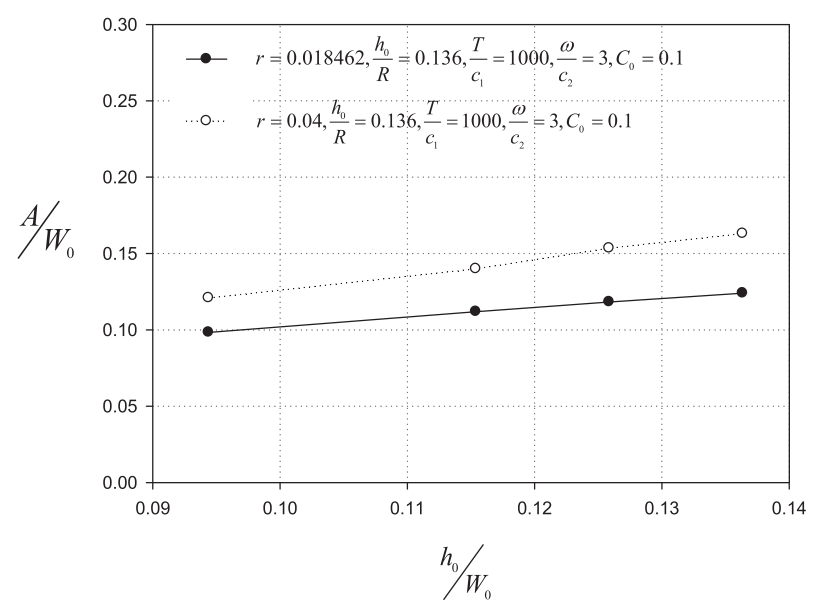

Fig. 9. The effect of $\frac{h_{0}}{W_{0}}$ on $\frac{A}{W_{0}}$.

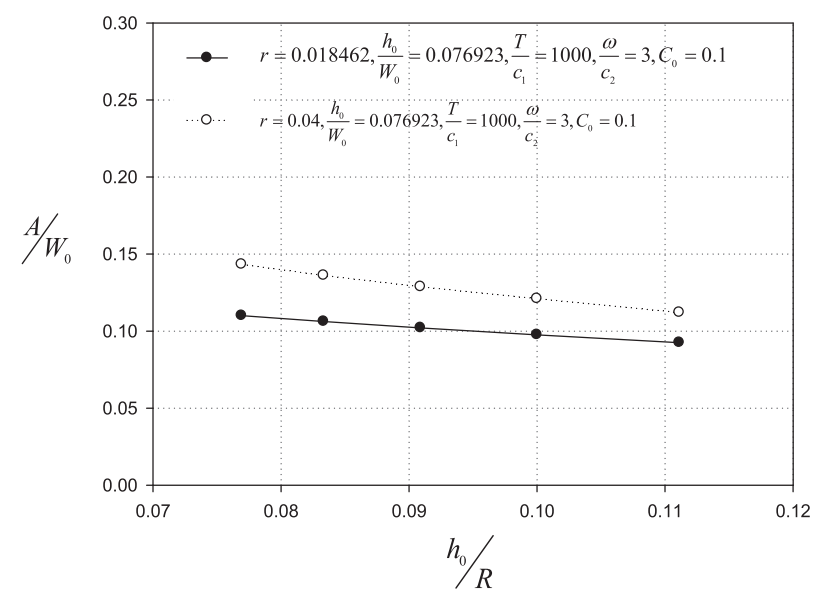

Fig. 10. The effect of $\frac{h_{0}}{R}$ on $\frac{A}{W_{0}}$.

The prediction error of the above equation, which is valid within the range of rolling conditions given in Table 1 , is found to be less than 10 percent, as shown in Fig. 11.

\section{A Model for the Prediction of Roll Force in Edge Rolling}

The actual roll force $F$ per unit thickness of the slab for

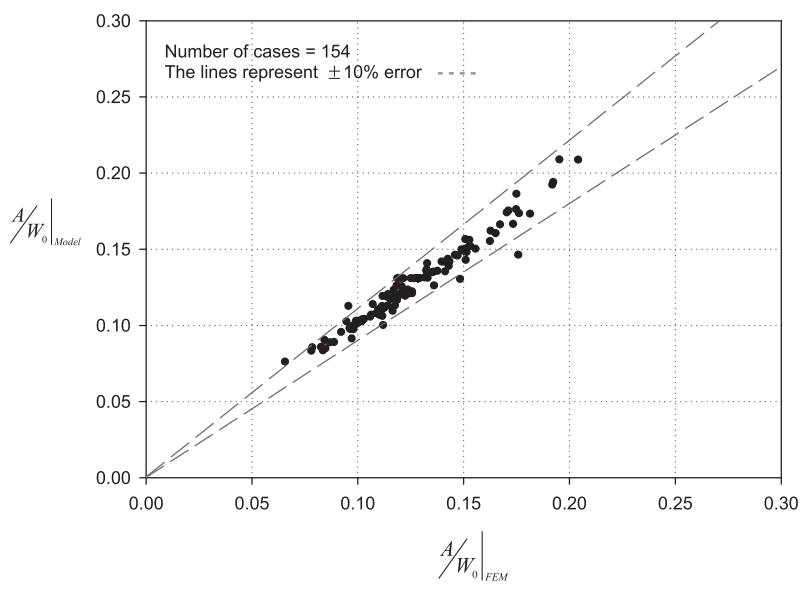

Fig. 11. The values of $\frac{A}{W_{0}}$, comparison between theory and FEM.

plain carbon steel may be represented in a form similar to Eq. (44), or

$$
F=\pi\left(\mu, C_{0}, T, \omega, R, W_{0}, W_{E}, h_{0}\right)
$$

Suppose that, in actual rolling, the width of a slab is reduced from $2 W_{0}$ to $2 W_{E}$. Now, for the same rolling geometry and the same slab consider a hypothetical mode of rolling, as shown in Fig. 3. Further, assume that the temperatures remain constant during rolling. Then,

We may define roll force $F^{\prime}$, for ideal rolling in which each segment of the slab is uniformly compressed under the plane strain condition while passing through the roll bite, and no friction is present at the roll-slab interface. The ideal roll force $F^{\prime}$ per unit thickness of the slab, may be given by

$$
\begin{aligned}
& F^{\prime}=\frac{2}{\sqrt{3}} \int_{0}^{\phi_{1}} \bar{\sigma}(\bar{\varepsilon}, \dot{\bar{\varepsilon}}, T) R \cos \phi d \phi \\
& \phi_{1}=\sin ^{-1}\left(\frac{l}{R}\right) \\
& \bar{\varepsilon}=-\frac{2}{\sqrt{3}} \ln \frac{W}{W_{E}} \\
& \dot{\bar{\varepsilon}}=\frac{2}{\sqrt{3}} \frac{\tan \phi}{W^{2}(\phi)} R \omega W_{E} \\
& W(\phi)=R(1-\cos \phi)+W_{E}
\end{aligned}
$$

where $\phi_{1}$ is bite angle.

From Eqs. (48) and (49), the actual roll force may be expressed in a dimensionless form

$$
\frac{F}{F^{\prime}}=\tilde{\pi}\left(\mu, C_{0}, \frac{T}{c_{1}}, \frac{\omega}{c_{2}}, s, r, t\right) \ldots
$$

where

$$
\begin{gathered}
s=\frac{l_{d}}{W_{0}+W_{E}} \\
r=\frac{W_{0}-W_{E}}{W_{0}} \\
t=\frac{W_{0}}{h_{0}} \ldots .
\end{gathered}
$$




$$
l_{d}=\sqrt{2 R\left(W_{0}-W_{E}\right)}
$$

where $l_{d}$ is the bite zone length. Note that $c_{1}$ and $c_{2}$ are introduced to render $T$ and $\omega$ dimensionless, respectively. In this paper, $c_{1}=1{ }^{\circ} \mathrm{C}$ and $c_{2}=1 \mathrm{rad} / \mathrm{sec}$ are selected.

From a series of FE simulation, it was revealed that $\frac{F}{F^{\prime}}$ is gravely affected by the dimensionless parameters describing the rolling geometry and friction, but very little by other parameters. It follows that, assuming that for $\mu=0.3$, which is a close approximation of the actual friction coefficient for hot rolling,

$$
\frac{F}{F^{\prime}}=\tilde{\pi}(s, r, t)
$$

A mathematical formula may be derived from the regression of the data obtained from a series of FE simulation. The result is,

$$
\begin{aligned}
\frac{F}{F^{\prime}}= & 10.783-0.3488 t-63.43 r-127.914 s+0.0057 t^{2}+4.28 r t \\
& -157.311 r^{2}+4.775 s t+1629.363 s r+479.008 s^{2}-0.07843 r t^{2} \\
& -1.3701 r^{2} t-0.07563 s t^{2}-86.7533 s r t-4057.4 s r^{2} \\
& -16.9924 s^{2} t-8127.533 s^{2} r+0.01633 r^{2} t^{2}+1.5326 s r t^{2} \\
& +330.382 s r^{2} t+0.2543 s^{2} t^{2}+361.6 s^{2} r t+33672.364 s^{2} r^{2} \\
& -6.035 s r^{2} t^{2}-5.98 s^{2} r t^{2}-1803.416 s^{2} r^{2} t+30.977 s^{2} r^{2} t^{2}
\end{aligned}
$$

The prediction error of the above equation, which is valid within the range of rolling conditions described by $s=$ $0.045-0.24, r=0.005-0.095$, and $t=4-40$, is found to be less than 5 percent.

\section{Numerical Procedure}

With all the development so far, the functional $\phi\left(u_{i}^{*}\right)$ to be minimized (see Eq. (1)) has only two unknown variables, that is, $\alpha$ and $h_{r}$, since $A$ is given by Eq. (47), and $h_{b}$ is given by Eq. (36), as a function of $h_{r}$. With $\alpha=8$ being selected for the present investigation, our model is reduced to be a problem of finding the optimal value of $h_{r}$ which minimizes $\phi\left(u_{i}^{*}\right)$.The optimization problem may be solved by a technique known as quadratic curve fitting, ${ }^{10)}$ as follows.

Step-1. Let the function to be minimized be $f(d)$. Set a sufficiently large range for $d$, that is, assume $d_{L}$ and $d_{U}, d_{L}<$ $d<d_{U}$.

Step-2. Let $d_{i}$ be an intermediate point in the interval $d_{L}<$ $d<d_{U}$. Calculate $f\left(d_{i}\right), f\left(d_{L}\right)$, and $f\left(d_{U}\right)$.

Step-3. Compute the coefficients $a_{0}, a_{1}, a_{2}$ of the quadratic function $q(d)=a_{0}+a_{1} d+a_{2} d^{2}$ which passes through the three points found in Step-2. Compute $\bar{d}=-a_{1} / 2 a_{2}$ at which $q(d)$ is minimized. Also, compute $f(\bar{d})$.

Step-4. Let us consider the case in which $d_{i}<\bar{d}$ and $f\left(d_{i}\right)<f(\bar{d})$ (All the other cases are not considered here, since they are treated essentially in the same manner). Then, obviously, $d^{*}$, the point at which the minimum value of $f(d)$ is achieved is $d_{L}<d^{*}<\bar{d}$. It is in this regard that an improved guess for $d_{L}, d_{U}, d_{i}$ may be made. That is, $d_{L}=d_{L}$, $d_{U}=\bar{d}$, and $d_{i}=d_{i}$.

Step-5. If two successive estimates of $f(\bar{d})$ are sufficient- ly close to each other, stop. The solution is $d^{*}=\bar{d}$. If not, go to Step-2.

For the calculation of $f(d)=\phi\left(u_{i}^{*}\right)$, all the integrals appearing in Eq. (1) are calculated numerically, using ten points Gauss Quadrature formula. With $h_{r}$ thus found, $h_{b}$ may be calculated from Eq. (36), using Eq. (47) for the estimation of the parameter $A$. Then, the dog bone shape may be obtained from Eqs. (17) and (19).

\section{Result and Discussion}

The values of $\frac{h_{b}}{h_{0}}$ as well as $\frac{h_{r}}{h_{0}}$ predicted by the model for various cases agree well with predictions from FE simulation, with the differences being within 5 percent, as illustrated in Fig. 12. The predicted dog-bone shapes are in good agreement with the predictions from FE simulation, as shown in Figs. 13 and 14. The predicted dog-bone shape also agrees favorably with the Shihabara's experiment, ${ }^{3)}$ as shown in Fig. 15, indicating the validity of either the present model or FEM. From these figures, it is seen that the dog-

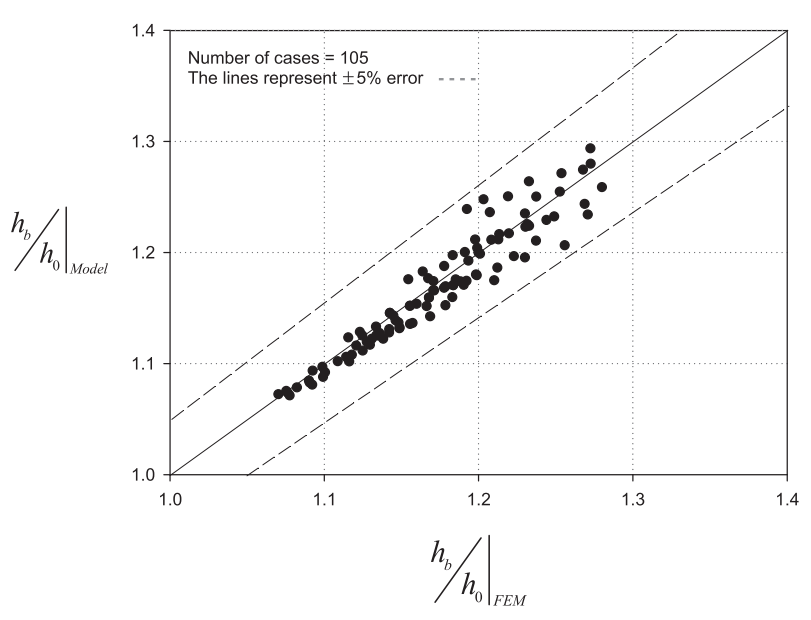

Fig. 12. The values of $\frac{h_{b}}{h_{0}}$, comparison between theory and FEM.

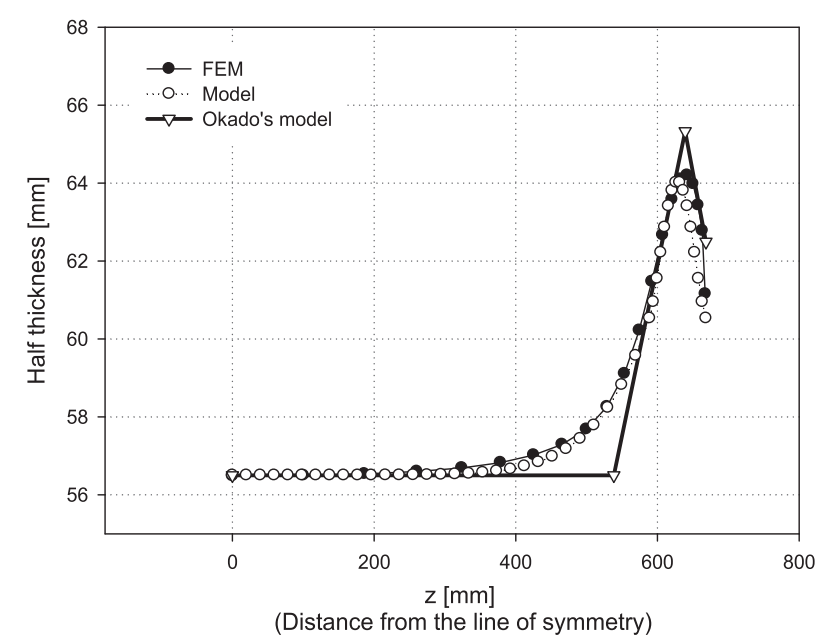

Fig. 13. The dog-bone shapes, theory and FEM. Entry slab width $=1375 \mathrm{~mm}$, exit slab width $=1337 \mathrm{~mm}$, slab thickness $=113 \mathrm{~mm}$, roll radius $=550 \mathrm{~mm}$, slab temperature $=$ $1000^{\circ} \mathrm{C}$, roll velocity $=3.091 \mathrm{rad} / \mathrm{sec}, 0.1 \%$ plain carbon steel. 


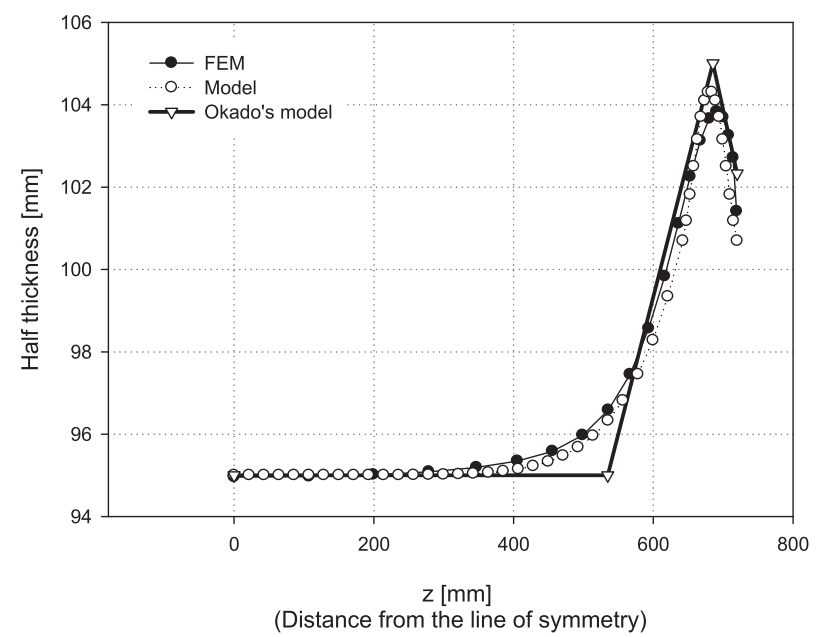

Fig. 14. The dog-bone shapes, theory and FEM. Entry slab width $=1470 \mathrm{~mm}$, exit slab width=1440 mm, slab thickness $=190 \mathrm{~mm}$, roll radius $=550 \mathrm{~mm}$, slab temperature $=$ $1000^{\circ} \mathrm{C}$, roll velocity $=3.091 \mathrm{rad} / \mathrm{sec}, 0.1 \%$ plain carbon steel.

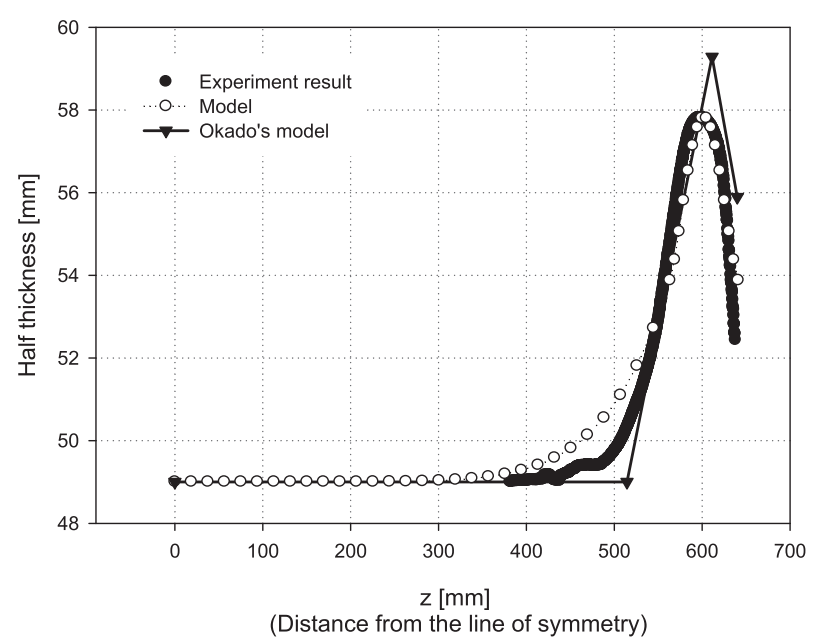

Fig. 15. The dog-bone shapes, comparison between theory and experiment of Shibahara et al. The rolling conditions for the experiment which are taken from Chapter 3 of reference 2 . They are, entry slab width $=1335 \mathrm{~mm}$, exit slab width $=1280 \mathrm{~mm}$, slab thickness $=98 \mathrm{~mm}$, and roll radius $=305 \mathrm{~mm}$. For other conditions which are not shown in the reference, but required by the present model, it is assumed that, slab temperature $=1000^{\circ} \mathrm{C}$, roll velocity $=3.0 \mathrm{rad} / \mathrm{sec}, 0.2 \%$ plain carbon steel, and $\mu=0.3$.

bone height and the dog-bone profile can be predicted with much higher level of accuracy than those predicted by Okado. $^{4)}$

It may be seen from Fig. 16 that $\frac{h_{b}}{h_{0}}$ increases linearly with the increase in reduction, as may be expected. It is found that $\frac{h_{b}}{h_{0}}$ also increases linearly with $\frac{h_{0}}{R}$, as shown in Fig. 17, indicating that the decrease in the dog-bone height may be achieved by increasing the roll diameter. Regarding the effect of $\frac{h_{0}}{W_{0}}, \frac{h_{b}}{h_{0}}$ decreases with the increase in $\frac{h_{0}}{W_{0}}$, indicating that when $r$ and $\frac{h_{0}}{R}$ are constant, the dog-bone height increases with the increase in the slab width.

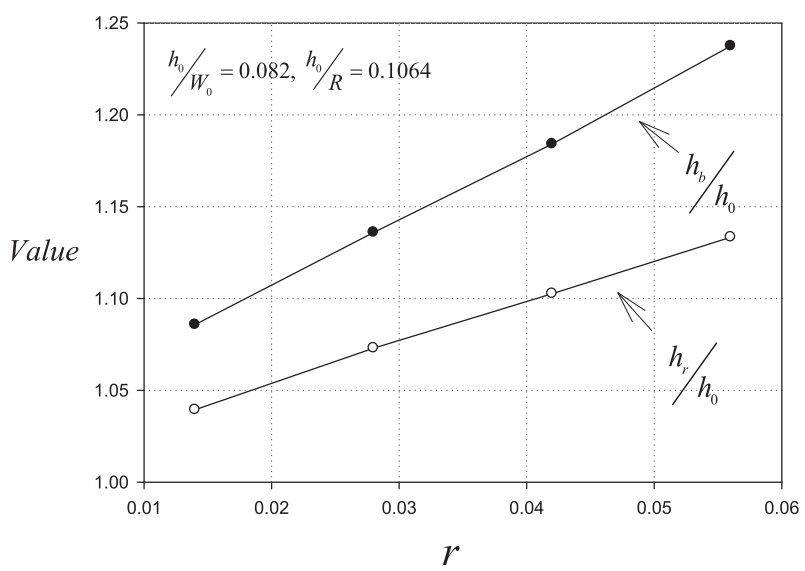

Fig. 16. The effect of $r$ on $\frac{h_{b}}{h_{0}}$.

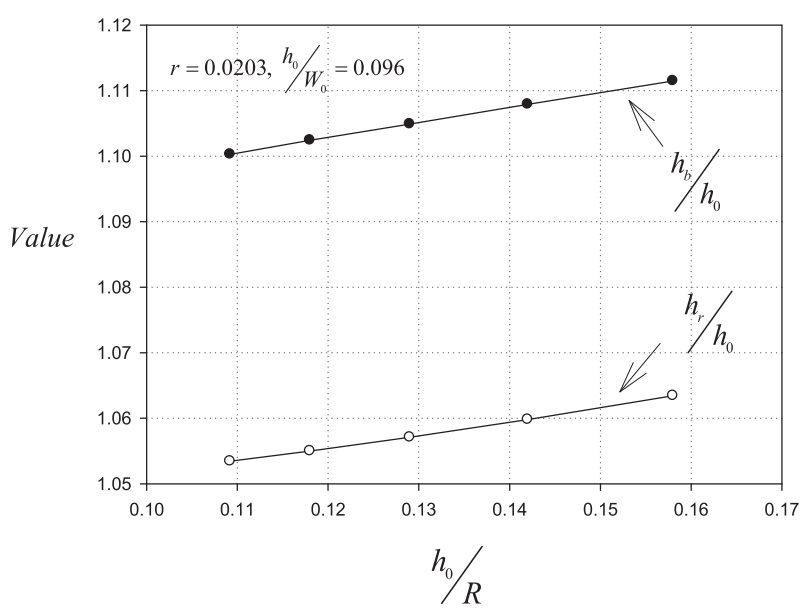

Fig. 17. The effect of $\frac{h_{0}}{R}$ on $\frac{h_{b}}{h_{0}}$.

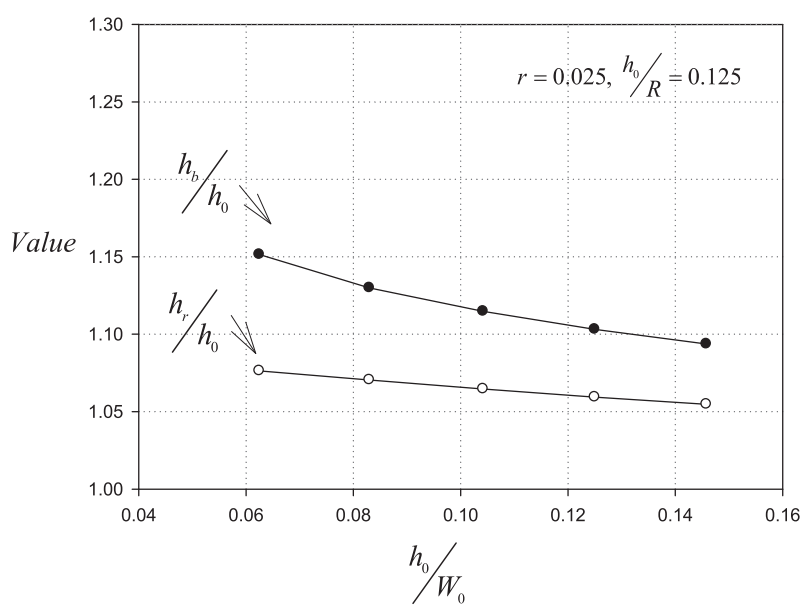

Fig. 18. The effect of $\frac{h_{0}}{W_{0}}$ on $\frac{h_{b}}{h_{0}}$.

\section{Concluding Remarks}

Precision control of the width of slabs, plates and strips, is vital for product quality and production economy in steel mills. A common practice in the production line is to reduce the width in the roughing mill by performing vertical rolling 
as well as horizontal rolling. However, the formation of so called 'dog-bone' at the edge of the slab would affect the final width after horizontal rolling that follows, and therefore, it is vital to predict the dog-bone shape precisely for sound width control. The model presented through this investigation is developed rigorously on the basis of the scientific principle, and differs clearly from the existing models which are mostly empirical.

Comparison is made among Shibahara et al's experiment, FEM, Okado et al's model, and the present model. The results demonstrate the present model's remarkably enhanced level of accuracy with regard to predicting the dog-bone shape, compared to the conventional models. It is in this regard that the model proposed in this paper will play an important role as an effective tool in achieving sound process control in steel mills.

Yet, a need may be raised for improving the prediction accuracy of the present model, examining the end part of the dog-bone represented by function $h_{2}(x, z)$. It is expected that the difference between the prediction and the actual dogbone shape (or FEM) may be reduced by selecting a higher order, asymmetric function for $h_{2}(x, z)$, which is to be left as a future work.

\section{Nomenclature}

$F \quad=$ roll force per unit slab thickness

$h_{0} \quad=$ half thickness of the slab at roll entrance

$h(x, z)=$ half thickness of the slab at point $(\mathrm{x}, \mathrm{z})$ in the bite zone

$k=$ shear yield stress

$l=$ projected length of roll/slab contact arc

$R \quad=$ roll radius

$r \quad=$ width reduction ratio

$T=$ slab temperature

$V_{0} \quad=$ Inlet velocity of the slab

$\vec{u}_{t}^{*} \quad=$ tangential velocity of the slab at the roll-slab interface $\vec{u}_{t D} \quad=$ roll velocity

$u_{x}^{*}, u_{y}^{*}, u_{z}^{*}=$ components of the admissible velocity vector

$u_{x}, u_{y}, u_{z}=$ Components of the actual velocity vector

$W_{0} \quad=$ half width of the slab at roll entrance

$W_{E} \quad=$ half width of the slab at roll exit

$\bar{\varepsilon} \quad=$ effective strain, calculated from the actual velocity field

$\dot{\bar{\varepsilon}} \quad=$ effective strain rate, calculated from the actual velocity field

$\dot{\bar{\varepsilon}}^{*} \quad=$ effective strain rate, calculated from the admissible velocity field

$\mu \quad=$ coefficient of Coulomb friction

$\Omega \quad=$ volume representing the bite zone

$\Gamma_{c} \quad=$ roll-slab interface

$\Gamma_{l s} \quad=$ interface where velocity discontinuity occurs in the admissible velocity field

$\left|\overrightarrow{\Delta u}^{*}\right|=$ magnitude of the velocity discontinuity at $\Gamma_{l s}$

$\sigma_{n} \quad=$ normal stress

$\bar{\sigma} \quad=$ flow stress

$\omega=$ angular velocity of the roll

$\lambda=$ frictional stress

$\phi_{1} \quad=$ bite angle

\section{REFERENCES}

1) N. Tazoe, H. Honjyo, M. Takeuchi and T. Ono: AISE Spring Conf., AISE, Warrendale, PA, (1984).

2) V. B. Ginzburg: High-quality Steel Rolling Theory and Practice, Marcel Dekker, New York, (1993), 327.

3) T. Shibahara, Y. Misaka, T. Kono, M. Koriki and H. Takemoto: $J$. Iron Steel Inst. Jpn., 67 (1981), 2509.

4) M. Okado, T. Ariizumi, Y. Noma, K. Yabuughi and Y. Yamazaki: J. Iron Steel Inst. Jpn., 67 (1981), 2516.

5) S. Xiong, X. Liu, G. Wang and W. Zhang, ASM Int., 6 (1997), 757.

6) S. I. Oh and S. Kobayashi: Int. J. Mech. Sci., 17 (1975), 293.

7) V. Nagpal: Trans. ASME. J. Eng. Ind., 99 (1977), 624.

8) K. Komori: Sosei-to-Kako, 34 (1993), No. 394, 1226 (in Japanese).

9) T. H. Kim, W. H. Lee and S. M. Hwang: ISIJ Int., 43 (2003), 1947.

10) J. S. Arora: Introduction to Optimal Design, McGraw Hill, New York, (1989).

11) S. Shida: J. Jpn. Soc. Technol. Plast., 20 (1969), 610. 